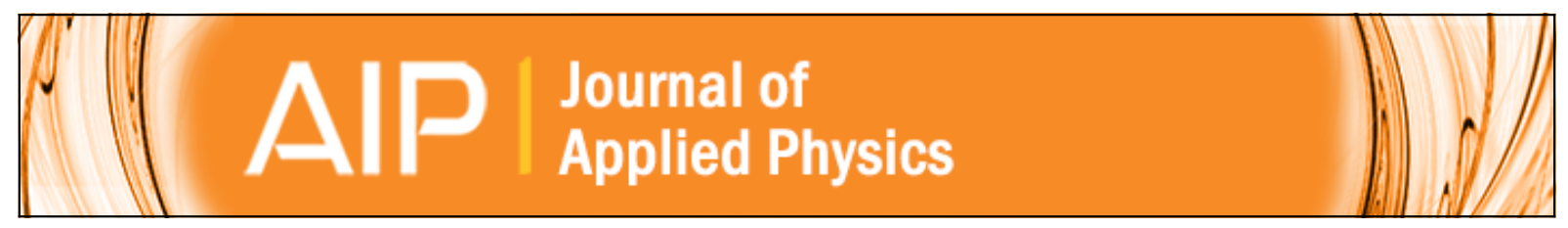

\title{
Effect of uniaxial stress on the large-signal electromechanical properties of electrostrictive and piezoelectric lead magnesium niobate lead titanate ceramics
}

D. Viehland, Jie-Fang Li, E. McLaughlin, J. Powers, R. Janus, and H. Robinson

Citation: Journal of Applied Physics 95, 1969 (2004); doi: 10.1063/1.1641960

View online: http://dx.doi.org/10.1063/1.1641960

View Table of Contents: http://scitation.aip.org/content/aip/journal/jap/95/4?ver=pdfcov

Published by the AIP Publishing

\section{Articles you may be interested in}

High temperature properties of $\mathrm{BiScO} 3-\mathrm{PbTiO} 3$ piezoelectric ceramics

J. Appl. Phys. 106, 014102 (2009); 10.1063/1.3158542

Influences of perpendicular compressive stress on the dielectric and ferroelectric properties of electrostrictive and piezoelectric $\mathrm{Pb}$ ( Mg $1 / 3 \mathrm{Nb} 2$ / 3 ) O 3 - $\mathrm{PbTiO} 3$ ceramics

J. Appl. Phys. 104, 034101 (2008); 10.1063/1.2960518

Piezo-, pyro-, ferro-, and dielectric properties of ceramic/polymer composites obtained from two modifications of lead titanate

J. Appl. Phys. 97, 034104 (2005); 10.1063/1.1847727

Effects of ferroelectric switching on the piezoelectric small-signal response (d 33 ) and electrostriction (M 33 ) of lead zirconate titanate thin films

J. Appl. Phys. 95, 4976 (2004); 10.1063/1.1690098

Intrinsic and coupling-induced elastic nonlinearity of lanthanum-doped lead magnesium niobate-lead titanate electrostrictive ceramic

Appl. Phys. Lett. 77, 1387 (2000); 10.1063/1.1290268

\section{MIT LINCOLN} LABORATORY CAREERS

Discover the satisfaction of innovation and service to the nation
- Space Control

- Air \& Missile Defense

- Communications Systems \& Cyber Security

- Intelligence, Surveillance and

Reconnaissance Systems
- Advanced
Electronics
- Tactical Systems
- Homeland
Protection
- Air Traffic Control

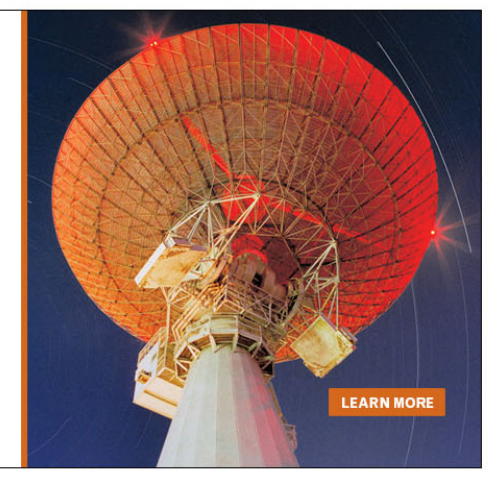




\title{
Effect of uniaxial stress on the large-signal electromechanical properties of electrostrictive and piezoelectric lead magnesium niobate lead titanate ceramics
}

\author{
D. Viehland ${ }^{\text {a) }}$ and Jie-Fang Li \\ Department of Materials Science and Engineering, Virginia Tech, Blacksburg, Virginia 24061
}

E. McLaughlin, J. Powers, R. Janus, and H. Robinson

Naval Sea Systems Command, Newport, Rhode Island 02841

(Received 20 August 2003; accepted 25 November 2003)

\begin{abstract}
The electromechanical performance characteristics of electrostrictive $0.9 \mathrm{~Pb}\left(\mathrm{Mg}_{1 / 3} \mathrm{Nb}_{2 / 3}\right) \mathrm{O}_{3}$ $-0.1 \mathrm{PbTiO}_{3}$ and piezoelectric $0.7 \mathrm{~Pb}\left(\mathrm{Mg}_{1 / 3} \mathrm{Nb}_{2 / 3}\right) \mathrm{O}_{3}-0.3 \mathrm{PbTiO}_{3}$ ceramics have been investigated under uniaxial stress $(\sigma)$. The results demonstrate that the large-signal electromechanical properties of electrostrictive ceramics are decreased with increasing $\sigma$, whereas those of the piezoelectric are increased but accompanied by significantly increased hysteretic losses. (C) 2004 American Institute of Physics. [DOI: 10.1063/1.1641960]
\end{abstract}

\section{INTRODUCTION}

$(1-x) \mathrm{Pb}\left(\mathrm{Mg}_{1 / 3} \mathrm{Nb}_{2 / 3}\right) \mathrm{O}_{3}-(x) \mathrm{PbTiO}_{3} \quad[\mathrm{PMN}-\mathrm{PT}(1$ $-x) / x]$ crystalline solutions have been investigated for applications in acoustic transduction. PMN-PT ceramics have been studied for electrostrictive applications $(x<0.2) .{ }^{1-4}$ Electrostrictive materials have slim-loop quadratic polarization versus electric field $(P-E)$ and strain versus electric field $(\varepsilon-E)$ responses, where $\varepsilon$ is proportional to $P^{2}$, rather than $E$ as in a piezoelectric material. Piezoelectric compositions of PMN-PT ceramics also exist at higher PT contents $(x>0.25){ }^{5-7}$ Piezoelectric materials are characterized by hysteretic $P-E$ (and $\varepsilon-E$ ) responses. The differences between the electrostrictive and piezoelectric compositions of PMN-PT are due to differences in the degree of long-range polar order. ${ }^{8}$ In the lower PT content specimens, long-range polar order does not develop, rather relaxor ferroelectric characteristics are observed. Relaxors have a strong frequency dispersive dielectric response, ${ }^{9-11}$ and polar clusters or nanodomains. ${ }^{12,13}$ In the higher PT content specimens, long-range polar order develops upon cooling through a ferroelectric phase transition and normal micron-sized ferroelectric domains exist. ${ }^{8}$

Recent investigations have focused on using PMN-PT as a high-energy density electroacoustic projector/source. ${ }^{14}$ The acoustic energy density is enhanced with increasing PT content, with the optimum performance occurring near the morphotropic phase boundary. ${ }^{5-7}$ Unfortunately, this enhanced performance is accompanied by higher hysteretic losses. In acoustic transducer applications, ${ }^{15}$ uniaxial prestress inherently needs to be used. This is an important feature of electrostrictive and piezoelectric PMN-PT ceramics that needs to be investigated. Currently, it is not known how these materials will perform under conditions required for acoustic transducer design, which has an upper field limit in

${ }^{\text {a)} E l e c t r o n i c ~ m a i l: ~ v i e h l a n d @ m s e . v t . e d u ~}$ the vicinity of $15 \mathrm{kV} / \mathrm{cm}$ and uniaxial stress requirements of $\sim 5 \times 10^{7} \mathrm{~N} / \mathrm{m}^{2}$.

The purpose of this investigation was to study the effects of uniaxial stress on the large-signal electrostrictive and piezoelectric properties of PMN-PT ceramics. Investigations have been performed using $P-E$ and $\varepsilon-E$ methods.

\section{EXPERIMENTAL PROCEDURE}

Polycrystalline $0.7 \mathrm{~Pb}\left(\mathrm{Mg}_{1 / 3} \mathrm{Nb}_{2 / 3}\right) \mathrm{O}_{3}-0.3 \mathrm{PbTiO}_{3}$ (designated as 0.7 PMN-0.3 PT, or piezoelectric PMN-PT) and $0.9 \mathrm{~Pb}\left(\mathrm{Mg}_{1 / 3} \mathrm{Nb}_{2 / 3}\right) \mathrm{O}_{3}-0.1 \mathrm{PbTiO}_{3}$ (designated as 0.9 PMN-0.1 PT, or electrostrictive PMN-PT) materials were fabricated from powders formed using the columbite precursor method. ${ }^{16}$ Barlike specimens were cut into dimensions of $\sim 0.4 \mathrm{~cm} \times 0.4 \mathrm{~cm} \times 1.2 \mathrm{~cm}$. All specimens were electroded with gold on the ends of the bars. The piezoelectric 0.7 PMN-0.3 PT bars were then poled to a full remanent state prior to testing, however the electrostrictive $0.9 \mathrm{PMN}-0.1$ PT bars are relaxor ferroelectrics, and cannot be remanently poled.

Unipolar $P-E$ measurements were made using a modified Sawyer-Tower bridge, by applying a dc bias of 6.5 $\mathrm{kV} / \mathrm{cm}$ and a superimposed ac drive of $13 \mathrm{kV} / \mathrm{cm}$. The electrostrictive PMN-PT is nonremanent, so the zero point of the polarization is its natural state under zero field. The zero point of the piezoelectric 0.7 PMN-0.3 PT was determined by measuring the saturation polarization $\left(P_{s}=0.34 \mathrm{C} / \mathrm{m}^{2}\right)$ under a bipolar drive of $13 \mathrm{kV} / \mathrm{cm}$ and zero applied uniaxial stress. The $P-E$ data for the piezoelectric 0.7 PMN-0.3 PT under uniaxial stress was then referenced to this value of $P_{s}$. In addition, $\varepsilon-E$ measurements were simultaneously performed using a strain gauge. Strain gauges were mounted on the side of the bars and a mechanical load was applied along the length of the bar using a pneumatic cylinder. These measurements were performed using a drive frequency of $10 \mathrm{~Hz}$ at maximum applied field strengths of $13 \mathrm{kV} / \mathrm{cm}$. Measurements were performed as a function of mechanical prestress between 0 and $9 \times 10^{7} \mathrm{~N} / \mathrm{m}^{2}$. 


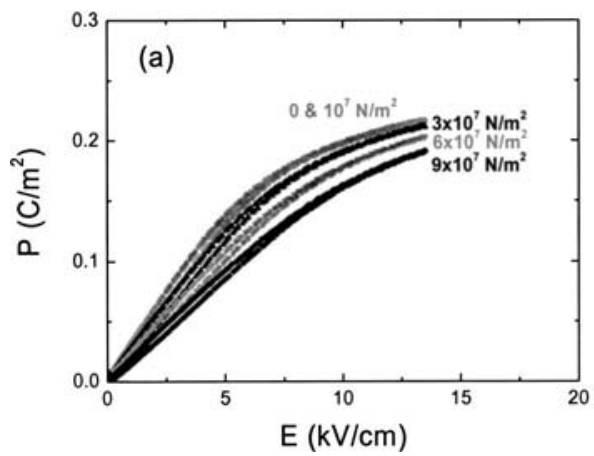

III. RESULTS

\section{A. Dependence of $P-E$ and $\varepsilon-E$ responses on $\sigma$}

Figure 1(a) shows the $P-E$ response of an electrostrictive $0.9 \mathrm{~Pb}\left(\mathrm{Mg}_{1 / 3} \mathrm{Nb}_{2 / 3}\right) \mathrm{O}_{3}-0.1 \mathrm{PbTiO}_{3}$ ceramic taken under unipolar drive at various $\sigma$. Figure 1(a) reveals a decrease in $P$ with increasing $\sigma$ for $E<13 \mathrm{kV} / \mathrm{cm}$. For example, $P$ at 13 $\mathrm{kV} / \mathrm{cm}, P_{(13 \mathrm{kV} / \mathrm{cm})}$, decreased from $\sim 0.22 \mathrm{C} / \mathrm{m}^{2}$ under small $\sigma$ to $\sim 0.18 \mathrm{C} / \mathrm{m}^{2}$ for $\sigma=9 \times 10^{7} \mathrm{~N} / \mathrm{m}^{2}$. Figure 1(b) shows the $\varepsilon-E$ response under various $\sigma$. Figure 1(b) also shows a decrease in $\varepsilon$ with increasing $\sigma$. The value of $\varepsilon$ under 13 $\mathrm{kV} / \mathrm{cm}, \varepsilon_{(13 \mathrm{kV} / \mathrm{cm})}$, decreased from $\sim 9 \times 10^{-4}$ under small $\sigma$ to $\sim 7 \times 10^{-4}$ for $\sigma=9 \times 10^{7} \mathrm{~N} / \mathrm{m}^{2}$. For electrostrictive PMN-PT ceramics, these results clearly demonstrate a suppression of the induced polarization and strain with increasing $\sigma$.

Figure 2(a) shows the $P-E$ response for a piezoelectric $0.7 \mathrm{~Pb}\left(\mathrm{Mg}_{1 / 3} \mathrm{Nb}_{2 / 3}\right) \mathrm{O}_{3}-0.3 \mathrm{PbTiO}_{3}$ ceramic taken under unipolar drive at various $\sigma$. Figure 2(a) reveals a significant change in $P_{r}$ with increasing $\sigma$. The value of $P_{r}$ increased from $\sim 0.26 \mathrm{C} / \mathrm{m}^{2}$ under small loads to $\sim 0.16 \mathrm{C} / \mathrm{m}^{2}$ under $6 \times 10^{7} \mathrm{~N} / \mathrm{m}^{2}$. Figure 2(b) shows the $\varepsilon-E$ response under various $\sigma$. Figure 2(b) demonstrates a decrease in the remanent $\varepsilon\left(\varepsilon_{r}\right)$ with increasing $\sigma$. The value of $\varepsilon_{r}$ decreased from $\sim 7.5 \times 10^{-4}$ under small $\sigma$ to approximately zero un$\operatorname{der} \sigma=6 \times 10^{7} \mathrm{~N} / \mathrm{m}^{2}$.

For piezoelectric PMN-PT ceramics, the data in Fig. 2(a) show that $P_{r}$ decreases with increasing $\sigma$, indicating a partial breakdown of ferroelectric order under $\sigma$. A significant increase in hysteretic loss was found to accompany these changes. Also, an increase in the slopes of the $\varepsilon-E$ curves with increasing $\sigma$ was found for $E<5 \mathrm{kV} / \mathrm{cm}$, however, for $E>5 \mathrm{kV} / \mathrm{cm}$, the slopes of the $\varepsilon-E$ curves were nearly the same at all $\sigma$ investigated. However, the electrostrictive ceramics did not exhibit stress-induced changes in $P_{r}$. This is because electrostrictive PMN-PT is a relaxor ferroelectric. ${ }^{8-13}$ Upon removal of $E$, relaxors inherently thermally depole into a polar cluster or nanodomain state. $^{8,12,13}$

\section{B. Dependence of the large-signal $d_{33}$ on $\sigma$}

The large-signal longitudinal piezoelectric constant $\left(d_{33}\right)$ can be calculated from the average slope of the $\varepsilon-E$ curve, i.e., $d_{33}=\Delta \varepsilon / \Delta E$. This is a linear approximation which, accordingly, has a limitation but is often used for materials performance estimation in transducer applications. Figure 3 shows the large-signal $d_{33}$ as a function of $\sigma$ for the electrostrictive and piezoelectric PMN-PT ceramics.

The value of the large signal $d_{33}$ can be seen to decrease with increasing $\sigma$ for the electrostrictive PMN-PT. For $\sigma$ $<2 \times 10^{7} \mathrm{~N} / \mathrm{m}^{2}, d_{33}$ was relatively independent of $\sigma$. However, as $\sigma$ was further increased, $d_{33}$ varied between 750 $\mathrm{pC} / \mathrm{N}$ at $\sigma=0$ to $600 \mathrm{pC} / \mathrm{N}$ at $\sigma=9 \times 10^{7} \mathrm{~N} / \mathrm{m}^{2}$. This decrease was due to the decrease in $\varepsilon$ with increasing $\sigma$, as shown in Fig. 1(b). However, for piezoelectric PMN-PT, the value of $d_{33}$ can be seen to increase with increasing $\sigma$. The value of $d_{33}$ varied between $500 \mathrm{pC} / \mathrm{N}$ at $\sigma=0 \mathrm{~N} / \mathrm{m}^{2}$ to 1100 $\mathrm{pC} / \mathrm{N}$ at $\sigma=9 \times 10^{7} \mathrm{~N} / \mathrm{m}^{2}$.

The results in Fig. 3 show that for $\sigma<2 \times 10^{7} \mathrm{~N} / \mathrm{m}^{2}$ (which is a modest stress for transducer design) that the large-signal $d_{33}$ is higher for the electrostrictive PMN-PT, relative to the piezoelectric one. For the piezoelectric ceramic, the pronounced increase of $d_{33}(>1000 \mathrm{pC} / \mathrm{N})$ with increasing $\sigma$ has promise for electromechanical applications. However, this increase in $d_{33}$ was accompanied by a signifi-
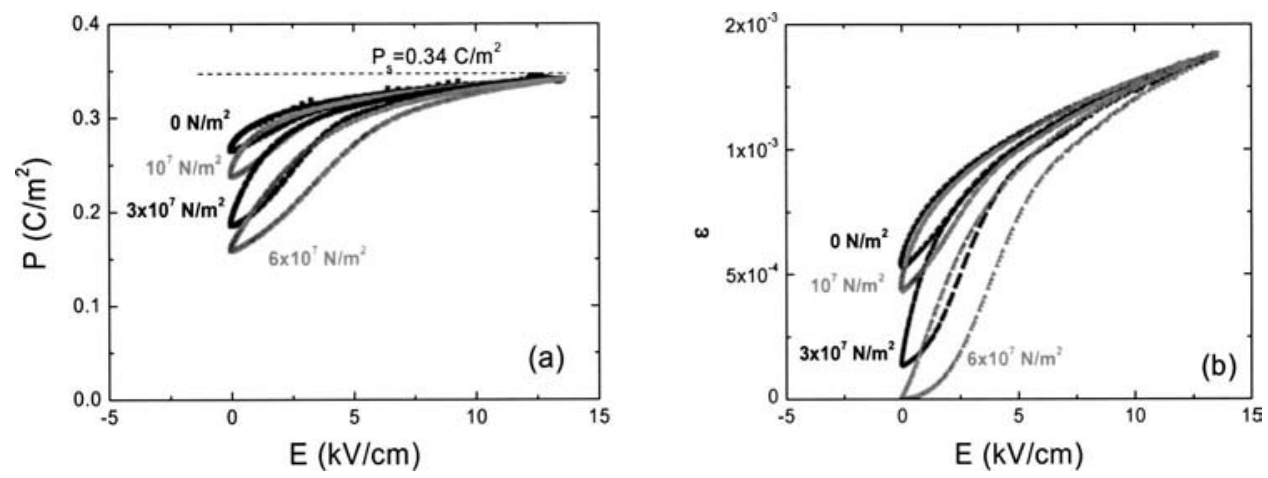

FIG. 2. Unipolar $P-E$ and $\varepsilon-E$ responses for piezoelectric PMN-PT ceramics as a function of uniaxial stress between 0 and $9 \times 10^{7} \mathrm{~N} / \mathrm{m}^{2}$. (a) $P-E$ response, and (b) $\varepsilon-E$ response. 


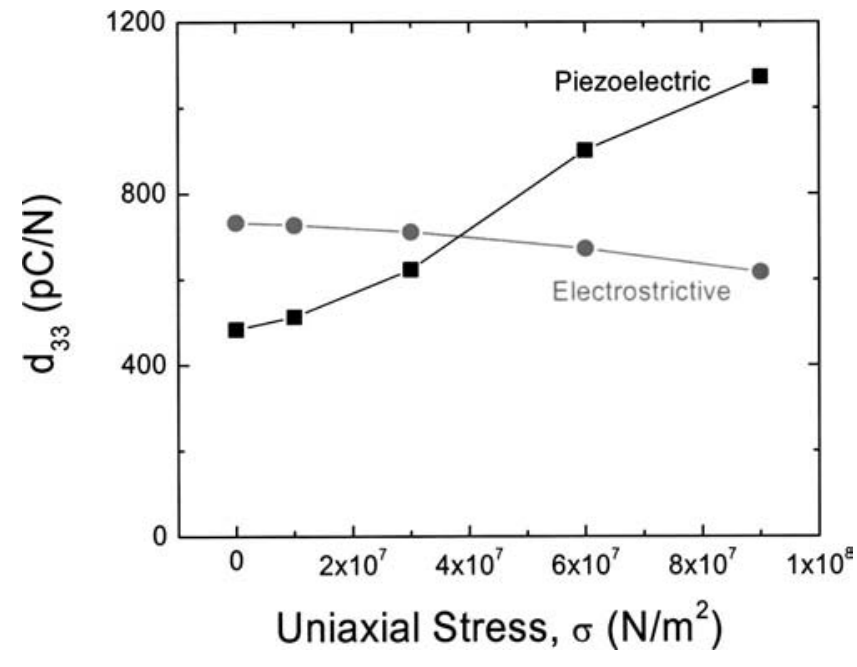

FIG. 3. Large-signal longitudinal piezoelectric constant $\left(d_{33}\right)$ as a function of $\sigma$ for electrostrictive PMN-PT and piezoelectric PMN-PT ceramics.

cant increase in hysteretic loss factor. The hysteretic energy loss was estimated by integrating the area of the $P-E$ loops using Greene's theorem. The loss factor was then calculated by dividing the energy loss by the stored electrical energy $1 / 2 P E$. Figure 4 shows the hysteretic loss factor as a function of $\sigma$ for electrostrictive and piezoelectric PMN-PT. The loss factor of the piezoelectric PMN-PT is much higher than for the electrostrictive, in addition the hysteretic loss of piezoelectric PMN-PT increased significantly with increasing $\sigma$.

The results presented in Fig. 4 for the hysteretic loss factor of piezoelectric PMN-PT ceramics clearly illustrate an upper limit of the high-power drive conditions. It is important to note that these calculations of the loss factor were determined for operating loops of a transducer material biased at $6.5 \mathrm{kV} / \mathrm{cm}$ and drove between zero and $13 \mathrm{kV} / \mathrm{cm}$. Other drive conditions would result in different loss factors. The advantages of higher piezoelectric constants are offset by problems in thermal stability, similar to that for soft PZT piezoelectric ceramics. However, the hysteretic loss for the electrostrictive PMN-PT does not strictly impose such limitations on high-power applications at lower drive frequencies. Recent investigations have shown that the hysteretic losses of electrostrictive PMN-PT can be further reduced under high-power drive by improved process control and substituent modification. ${ }^{17}$

\section{Dependence of acoustic energy density on $\sigma$} as: ${ }^{18,19}$

By convention, the acoustic energy density is defined

$$
\begin{aligned}
E_{\text {elastic }} & =1 / 2 Y_{1} \varepsilon_{\mathrm{rms}}^{2}=1 / 4 Y_{1} \varepsilon^{2}, \\
& =1 / 4 Y_{1} d_{33}^{2} E^{2},
\end{aligned}
$$

where $Y_{1}$ is Young's modulus. The acoustic energy density of the standard PZT-8 $\left(E_{\text {PZT- } 8}\right)$ is calculated by assuming a linear piezoelectric response over the operational field range, i.e., $\varepsilon=d_{33} E$. The values of $d_{33}$ and $Y_{1}$ for PZT-8 are equal

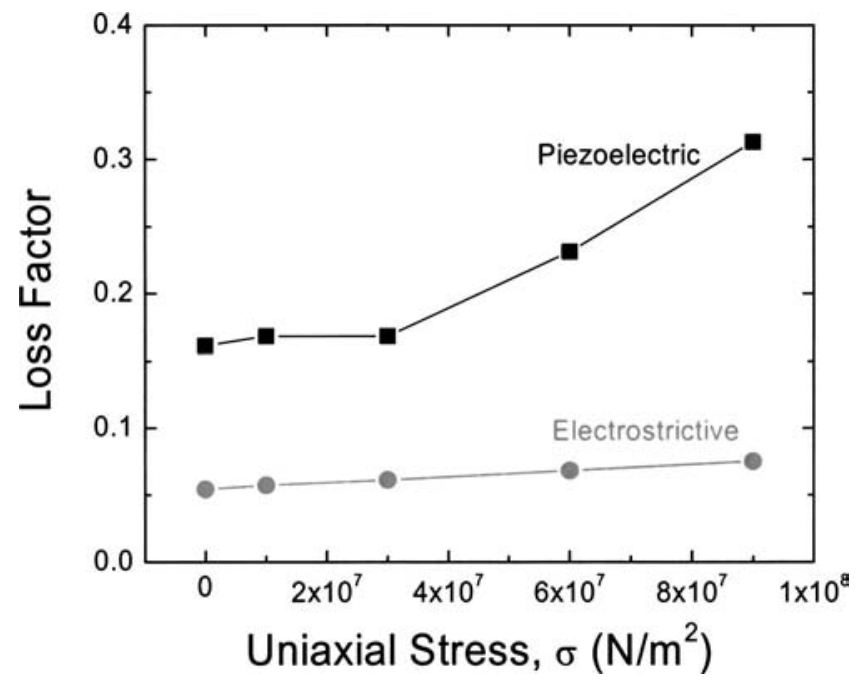

FIG. 4. Hysteretic loss as a function of $\sigma$ for electrostrictive PMN-PT and (b) piezoelectric PMN-PT ceramics.

to $2.25 \times 10^{-10} \mathrm{C} / \mathrm{N}$ and $7.4 \times 10^{10} \mathrm{~N} / \mathrm{m}^{2}$. Thus, the value of $E_{\text {PZT-8 }}$ can be estimated as $2108 \mathrm{~J} / \mathrm{m}^{3}$ for $E=13 \mathrm{kV} / \mathrm{cm}$.

Figure 5 shows the acoustic energy density (in $\mathrm{J} / \mathrm{m}^{3}$ ) as a function of $\sigma$ for electrostrictive and piezoelectric PMN-PT. For electrostrictive PMN-PT, the acoustic energy density can be seen to decrease with increasing $\sigma$. The energy density is $2.5 \times 10^{4} \mathrm{~J} / \mathrm{m}^{3}$ for $\sigma<2 \times 10^{7} \mathrm{~N} / \mathrm{m}^{2}$, whereas at $\sigma$ $=9 \times 10^{7} \mathrm{~N} / \mathrm{m}^{2}$ it is $1.5 \times 10^{4} \mathrm{~J} / \mathrm{m}^{3}$. For piezoelectric PMNPT, the acoustic energy density increases dramatically with increasing $\sigma$. The energy density is $8 \times 10^{3} \mathrm{~J} / \mathrm{m}^{3}$ under small $\sigma$, whereas it is $4.5 \times 10^{4} \mathrm{~J} / \mathrm{m}^{3}$ at $\sigma=9 \times 10^{7} \mathrm{~N} / \mathrm{m}^{2}$. Unfortunately, this significant increase in energy density is accompanied by a significant increase in hysteretic loss, as discussed above.

\section{Dependence of the large-signal electromechanical coupling coefficient on $\sigma$}

The electromechanical coupling coefficient $\left(k_{33}\right)$ at various uniaxial loads can be calculated as;

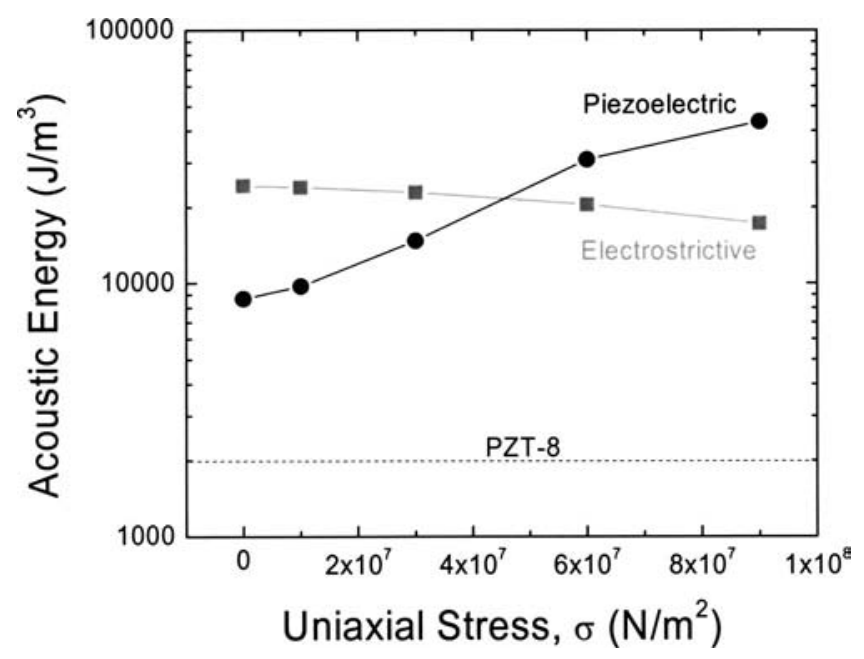

FIG. 5. Acoustical energy density as a function of $\sigma$ for electrostrictive PMN-PT and piezoelectric PMN-PT ceramics. 


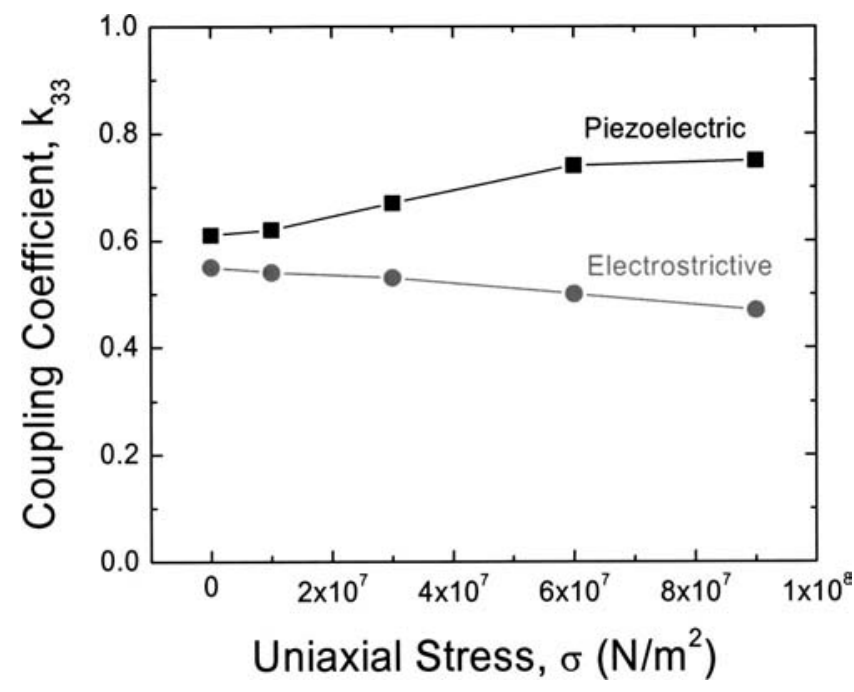

FIG. 6. Large-signal coupling coefficient $\left(k_{33}\right)$ as a function of $\sigma$ for electrostrictive PMN-PT and piezoelectric PMN-PT ceramics.

$$
k_{33}^{2}=d_{33}^{2} Y_{1} /\left(\varepsilon_{0} K\right),
$$

where $\varepsilon_{0}$ is the permittivity of free space $(8.85$ $\left.\times 10^{-12} \mathrm{~F} / \mathrm{m}\right), K$ is the relative dielectric constant, and $d_{33}$ is the longitudinal piezoelectric coefficient. $K$ can be approximated from the $P-E$ response over a quasi-linear range as $\delta P / \delta E$, and $d_{33}$ can be approximated over a quasi-linear range as $\delta \varepsilon / \delta E$, both of which have limitations. Quasi-linear approximations were performed for an $E_{\mathrm{dc}}$ of $8 \mathrm{kV} / \mathrm{cm}$ and an $E_{\mathrm{ac}}$ of $4 \mathrm{kV} / \mathrm{cm}$. The value of $Y_{1}$ can be determined from measurements of the $\varepsilon-\sigma$ response. Accordingly, $Y_{1}$ was determined at various dc bias levels $\left(E_{\mathrm{dc}}\right)$ by stress-strain $(\varepsilon-\sigma)$ measurements. The $\varepsilon-\sigma$ curves were linear and $Y_{1}$ was calculated from the slopes at $E_{\mathrm{dc}}=8 \mathrm{kV} / \mathrm{cm}$.

The value of $k_{33}$ as a function of $\sigma$ is shown in Fig. 6. For electrostrictive PMN-PT, $k_{33}$ can be seen to decrease with increasing $\sigma$ from $\sim 0.55$ at $\sigma=0 \mathrm{~N} / \mathrm{m}^{2}$ to $\sim 0.47$ at $\sigma$ $=9 \times 10^{7} \mathrm{~N} / \mathrm{m}^{2}$. For piezoelectric PMN-PT, $k_{33}$ can be seen to increase with increasing $\sigma$ from $\sim 0.60$ at $\sigma=0 \mathrm{~N} / \mathrm{m}^{2}$ to $\sim 0.75$ at $\sigma=9 \times 10^{7} \mathrm{~N} / \mathrm{m}^{2}$. The value of $k_{33}$ for hard PZT ceramics, conventionally used in high-power transducer applications, is $\sim 0.65$. $^{20}$

\section{DISCUSSION AND SUMMARY}

The uniaxial stress dependencies of electrostrictive and piezoelectric PMN-PT ceramics have been shown to be significantly different from each other. Electrostrictive PMN-PT has a higher large-signal $d_{33}$ and a higher acoustic energy density for $\sigma<2 \times 10^{7} \mathrm{~N} / \mathrm{m}^{2}$, but these values deteriorate with increasing $\sigma$ for $\sigma>2 \times 10^{7} \mathrm{~N} / \mathrm{m}^{2}$; whereas, the values of the large-signal $d_{33}$ and the acoustic energy density increase with increasing $\sigma$ for piezoelectric PMN-PT. This difference is due to a breakdown of ferroelectric order in piezoelectric PMN-PT with increasing $\sigma$, whereas electrostrictive PMN-PT naturally returns to a polar nanodomain state upon removal of $E$.

Electrostrictive PMN-PT has advantages over conventional "hard" PZT in acoustic energy density, but has reduced bandwidth. Piezoelectric PMN-PT has advantages in both acoustic energy density and bandwidth. A much higher acoustic energy density of $\sim 4.5 \times 10^{4} \mathrm{~J} / \mathrm{m}^{3}$ is achievable for piezoelectric PMN-PT ceramics under higher $\sigma$. However, to achieve this performance, unrealistic hysteretic losses need to be tolerated. The only way to reduce the hysteretic losses of piezoelectric PMN-PT is to use oriented crystals, which have been shown to have much lower losses and are close to those reported here for electrostrictive PMN-PT. ${ }^{21}$ Transducer designs should be able to rely on electrostrictive PMN-PT ceramics for $2 \times 10^{4} \mathrm{~J} / \mathrm{m}^{3}$ more energy density, a $k_{33}$ of 0.5 , and moderate losses under operational conditions of $\sigma<2 \times 10^{7} \mathrm{~N} / \mathrm{m}^{2}$. If higher coupling is required at this high-energy density and low loss factor, the only way to achieve it is by use of oriented PMN-PT piezoelectric crystals.

\section{ACKNOWLEDGMENTS}

This research was supported by the Office of Naval Research under Grant Nos. N000140210340, N000140210126, and MURI N000140110761.

${ }^{1}$ S. Jang, Ph.D. dissertation, The Pennsylvania State University, State College, 1979.

${ }^{2}$ G. Schmidt, Phase Transitions 20, 127 (1990).

${ }^{3}$ K. Uchino, Solid State Ionics 108, 43 (1998).

${ }^{4}$ W. Pan, Ph.D. dissertation, The Pennsylvania State University, State College, 1988.

${ }^{5}$ N. Kim, Ph.D. dissertation, The Pennsylvania State Univers, State College, 1989.

${ }^{6}$ T. Shrout, Z. Chang, N. Kim, and S. Markgraf, Ferroelectr., Lett. Sect. 12, 63 (1990).

${ }^{7}$ Y. H. Chen, D. Viehland, and K. Uchino, Jpn. J. Appl. Phys. , (2000).

${ }^{8}$ D. Viehland, M. Kim, Z. Xu, and J. Li, Appl. Phys. Lett. 67, 2471 (1995).

${ }^{9}$ G. Smolenskii and A. Agranovskaya, Sov. Phys. Solid State 1, 1429 (1960)

${ }^{10}$ J. Smith, Ph.D. dissertation, The Pennsylvania State University, State College, 1967.

${ }^{11}$ L. E. Cross, Ferroelectrics 76, 249 (1987).

${ }^{12}$ C. Randall, D. Barber, and R. Whatmore, J. Microsc. 45, 275 (1987).

${ }^{13}$ X. Dai, Z. Xu, and D. Viehland, Philos. Mag. B 70, 33 (1994).

${ }^{14}$ S. Pilgrim and S. Winzer (unpublished).

${ }^{15}$ D. Stansfield, Underwater Electroacoustic Transducers (Bath University Press, Bath, United Kingdom, 1991).

${ }^{16}$ S. Swartz and T. Shrout, Mater. Res. Bull. 17, 1245 (1982).

${ }^{17}$ D. Viehland and J. F. Li, J. Appl. Phys. 89, 1826 (2001).

${ }^{18}$ R. S. Wollett, J. Acoust. Soc. Am. 40, 1112 (1966).

${ }^{19}$ J. L. Butler (unpublished).

${ }^{20}$ B. Jaffe, W. Cook, and H. Jaffe, Piezoelectric Ceramics (Academic, New York, 1971).

${ }^{21}$ D. Viehland, J. Powers, and J. F. Li, J. Appl. Phys. 90, 2479 (2001). 